\title{
The Efficacy and Safety of Radiofrequency Catheter Ablation for Cardiac Arrhythmias in Pediatric Patients
}

\author{
Su Xinxing, ${ }^{1}$ Zhou Jie, ${ }^{2}$ Zhou Lu, ${ }^{2}$ He Bing ${ }^{2}$ \\ ${ }^{1}$ Department of Paediatrics, Yangzhou First People's Hospital, Yangzhou, China; ${ }^{2}$ Department of Paediatrics, Renmin Hospital of \\ Wuhan University, Wuhan, China
}

\section{ABSTRACT}

Background: We aimed to evaluate the acute and longterm efficacy and safety of radiofrequency catheter ablation (RFCA) in Chinese pediatric patients with arrhythmias.

Methods: We gathered clinical data from pediatric patients who underwent RFCA in several large medical centers in China between 2000 and 2019.

Results: A total of 4,622 different substrates in 4,622 patients were ablated, with the majority consisting of supraventricular tachycardia $(\mathrm{N}=3,831,82.9 \%)$ and ventricular arrhythmias (VAs) $(\mathrm{N}=791,17.1 \%)$. The majority of supraventricular tachycardia (SVT) consists of atrioventricular reentry tachycardia (AVRT) $(\mathrm{N}=2,492,65.0 \%)$ and atrioventricular nodal reentry tachycardia (AVNRT) $(\mathrm{N}=1,075,28.1 \%)$. The accessory pathway location was left-sided in 1,237(49.7\%) and right-sided in 1,251(50.3\%) of the 2,488 pathways. The VAs consist of premature ventricular contraction (PVC) $(\mathrm{N}=597,75.5 \%)$ and ventricular tachycardia (VT) (N = 194, 24.5\%). The duration of follow-up ranged from 1 month to 1 year. The overall acute/ long-term success rate of RFCA was 96.4\%/92.2\%, AVRT 97.8\%/93.5\%, AVRT 99.3\%/95.0, PVC 98.1\%/92.2\%, and VT $81.3 \% / 75.2 \%$. Serious complications occurred in 29 patients $(0.6 \%)$.

Conclusions: This present study shows that RFCA is effective and safe in the treatment of arrhythmias in Chinese pediatric patients, with a high success rate exceeding $90 \%$, low recurrence rate, and significantly reduces the risk of complications. The success rate of left-sided pathway ablation is slightly higher than that of the right-sided pathway. There is no difference in the success rate of RFCA among children of different ages. It could be the first-line therapy in the majority of pediatric patients with SVT and/ or VAs.

Received December 10, 2019; accepted February 17, 2020.

*The first two authors contribute equally to this work

Correspondence: He Bing, Department of Paediatrics, Renmin Hospital of Wuban University, Fiefang Road of Wuchang District, Wuban City, China, 430060; 86-027-88041911-81531 (e-mail: hebingwbu@163.com).

\section{INTRODUCTION}

Radiofrequency catheter ablation (RFCA) has been used for the treatment of arrhythmias in children since 1991 [Walsh 1991]. Both retrospective and prospective studies have showed that RFCA is an effective and safe way of arrhythmia treatment in pediatric patients with good efficacy [Calkins 1999; Van 2004; Lenarczyk 2009]. Recent studies have shown improved success rate and low complications by using updated techniques such as 3-dimensional mapping and navigation system and cryogenic energy [Kubuš 2014; Ceresnak 2015]. RFCA nowadays is considered the standard therapy for the majority of pediatric patients with supraventricular tachycardia (SVT). Although there are many single center and multicenter studies on radiofrequency ablation of arrhythmias in children, such data is less in China. The aim of this study was to evaluate the efficacy and safety of RFCA in the treatment of arrhythmias in pediatric patients in China.

\section{METHODS}

We gathered clinical data from pediatric patients $<18$ years of age who underwent RFCA in several large medical centers in China between 2000 and 2019. Data included age, gender, type of arrhythmia, acute success, duration of follow up, longterm success, complications, and the presence of congenital heart disease. We excluded patients who were unwilling to receive follow up. The RFCA indications referred to the expert consensus on RFCA in children [Friedman 2002]. A total of 4,622 ablation procedures were performed in 4,622 patients $(2,700$ male; 1,922 female $)<18$ years old. The age at first ablation was 7.8 years \pm 3.7 years. The weight of patients at ablation was $29.7 \mathrm{~kg} \pm 5.5 \mathrm{~kg}$. Diagnostic and ablation catheters used 4-6F and 5-7F, according to body size. For the substrate on the left side of the heart, retrograde approach or transseptal puncture was used. The evaluation of ablation endpoint is the same as the method reported in other study [Friedman 2002]. The Ensite Arry, Ensite NavX or Carto system and general temperature-controlled ablation catheter or cold saline perfusion ablation catheter were used. The duration of follow up ranged from 1 month to 1 year. Recurrence was defined as one or more of the following: symptoms of discomfort highly suggestive of recurrence and documentation of original arrhythmia. Acute success was defined for 


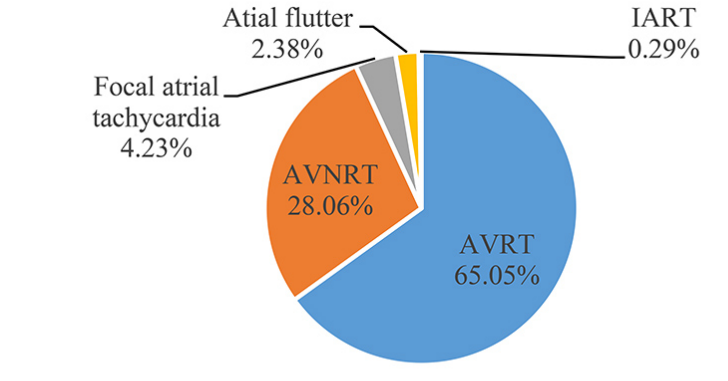

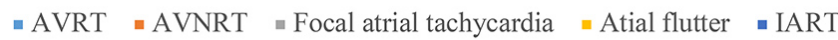

The proportion of different types of supraventricular tachycardia AVRT: atrioventricular reentry tachycardia, AVNRT: atrioventricular node reentry tachycardia, IART: intra-atrial reentry tachycardia.

each patient as elimination of inducible tachycardia in the electrophysiology lab and absence of tachycardia for 24 hours after radiofrequency ablation. Long-term success was defined as each patient living with no evidence of recurrent symptomatic arrhythmia within the follow-up interval. No patient was lost during the follow-up period.

Statistical analysis: Continuous data were expressed as mean \pm standard deviation. Difference in proportions between 2 groups were tested by the $\chi^{2}$ test. All statistical tests were two-sided, and a value $P<.05$ was regarded as significant. All statistical analysis was performed using SPSS 20.0 software.

\section{RESULTS}

Substrates: A total of 4,622 different substrates in 4,622 patients were ablated with the majority consisting of SVT (N $=3,831,82.9 \%)$ and ventricular arrhythmias (VAs) $(\mathrm{N}=791$, $17.1 \%$ ) (Table 1)

The majority of SVT consists of atrioventricular reentry tachycardia (AVRT) $(\mathrm{N}=2,492,65.0 \%)$ and atrioventricular nodal reentry tachycardia (AVNRT) $(\mathrm{N}=1,075$, $28.1 \%)$. The accessory pathway location was left-sided in $1,237(49.7 \%)$ and right-sided in 1,251 (50.3\%) of the 2,488 pathways. Other SVT included focal atrial tachycardia (N $=162,3.5 \%)$, atrial flutter $(\mathrm{N}=91,2 \%)$, and intra-atrial reentry tachycardia $(\mathrm{N}=11,0.2 \%)$ (Figure) The VAs consist of premature ventricular contraction (PVC) $(\mathrm{N}=597$, $75.5 \%)$ and ventricular tachycardia (VT) $(\mathrm{N}=194,24.5 \%)$. There were 455 VAs originated from the left side of the heart and 351 originated from the right side of the heart. Congenital heart disease (CHD) was present in 61 patients; the most common structural defects were atrial septal defect $(\mathrm{N}=19)$, ventrical septal defect $(\mathrm{N}=16)$, tetralogy of Fallot $(\mathrm{N}=7)$, Ebstein anomaly $(\mathrm{N}=6)$, dialated cardiomyopathy $(\mathrm{N}=4)$, patent ductus arteriosus $(\mathrm{N}=4)$, congenitally corrected transposition of great arteries $(\mathrm{N}=2)$ and double outlet right ventricle $(\mathrm{N}=2)$.

Acute and long-term success: The acute and long-term success rate of radiofrequency ablation for different types of arrhythmias are shown
Table 1. Patient characteristics

\begin{tabular}{lc}
\hline Baseline data & Total \\
\hline Patients & 4622 \\
Gender & $2700 \mathrm{M}, 1922 \mathrm{~F}$ \\
Weight $(\mathrm{kg})$ (mean \pm s.d.) & $29.7 \pm 5.5$ \\
Age of procedure (mean \pm s.d.) & $7.8 \pm 3.7$ \\
Arrthymogenic substrates & 4622 \\
SVT & 3831 \\
VAs & 791 \\
CHD & 61 \\
\hline
\end{tabular}

AVRT: atrioventricular reentry tachycardia; AVNRT: atrioventricular node reentry tachycardia; VAs: ventricular arrthymias; IART: intra-atrial reentry tachycardia; CHD: congenital heart disease

in Table 2. The overall acute/long-term success rate of radiofrequency ablation was $96.4 \% / 92.2 \%$, respectively, and the recurrence was $4.8 \%$. Among the SVT, the most common arrhythmias were AVRT with an acute/long-term success rate of $97.8 \% / 93.5 \%$, respectively, and the recurrence was $3.4 \%$. AVNRT with an acute/long-term success rate of $99.3 \% / 95.0 \%$, respectively, and the recurrence was $4.4 \%$. Among 806 ablated ventricular arrhythmias, the acute/long-term success rate of VAs were $94.4 \%$ and $88.2 \%$, respectively. The acute/long-term success rate of PVC and VT were $98.1 \% / 92.2 \%, 81.1 \% / 75.3 \%$, and the recurrence were $5.4 \%$ and $7.8 \%$, respectively.

The acute and long-term success rate of radiofrequency ablation for specific accessory pathway localization and children of different ages is shown in Table 3. In the subgroup of left-sided and right-sided accessory pathways, acute and longterm success rates were $97.8 \% / 96.9 \%$ and $93.5 \% / 89.8 \%$, respectively. In the subgroup of ages, the acute and long-term success rates for patients < or > 3 years old were $93.3 \% / 87.6$ and $97.1 \% / 92.5 \%$, respectively.

Complications: Serious complications occurred in 29 patients $(0.6 \%)$. Complete atrioventricular block occurred in 7 patients. The block was transient in 1 patient and the remaining 6 patients needed to be implanted with pacemakers. Twodegree atrioventricular block occurred in 7 patients and medical intervention was not needed; all returned to normal during the follow-up period. Among the substrates, AVNRT was more likely to have complications, due to the special anatomy. Vascular-related complications occurred in 15 patients. Femoral artery pseudoaneurysm and arteriovenous fistula requiring surgical revision occurred in 6 and 4 patients, respectively. Five patients with venous thromboembolism needed to receive oral anticoagulants. All patients returned to normal after symptomatic treatment.

\section{DISCUSSION}

Radiofrequency catheter ablation has been used in children with supraventricular tachycardia since 1991 [Blaufox 
Table 2. Acute and long-term success of RFCA

\begin{tabular}{lccc}
\hline Substrate & Acute success (\%) & Long-term success (\%) & $P$ \\
\hline All substrate & 96.4 & 92.2 & $>.05$ \\
AVRT & 97.8 & 93.5 & $>.05$ \\
AVNRT & 99.3 & 95.0 & $>.05$ \\
VAs & 94.4 & 88.2 & $>.05$ \\
Focal atrial tachycardia & 82.1 & 69.1 & $>.05$ \\
Atrial flutter & 95.6 & 85.7 & $>.05$ \\
IART & 100 & 100 & $>.05$ \\
\hline
\end{tabular}

AVRT: atrioventricular reentry tachycardia; AVNRT: atrioventricular node reentry tachycardia; VAs: ventricular arrthymias; IART: intra-atrial reentry tachycardia

1991]. RFCA of SVT and VAS in children is the recommended treatment for patients with severe symptoms, repeated recurrence, drug refractoriness and risk of sudden cardiac death [Rodriguez 1997; Brugada 2013]. This procedure has become first-line treatment for most patients with SVT, which can eliminate symptoms and improve the quality of life [Strieper 2010]. RFCA showed favorable efficacy and safety in patients with significant clinical symptoms and/ or drug-refractory arrhythmias [Blaufox 2005; Johnsrude 2008]. For smaller children $<18$ months, RFCA also showed good efficacy without increasing the risk of complications [Blaufox 2001].

The aim of this study was to analyze the main types of arrhythmias, efficacy, and safety of RFCA in children in China to better supply ablation data from a certain area. The most important criteria for RFCA in pediatric patients are success rates, major complications, and a low rate of tachycardia recurrence. The present study showed that the overall success rate of RFCA was $96.4 \%$, slightly higher than that of other pediatric reports (95.7\%-96.0\%) [Calkins 1999; Van 2004]. In accordance with other studies, the AVRT and AVNRT were the most common arrhythmogenic substrates [Lenarczyk 2009; Lee 2010]. The acute and long-term success rate of AVRT in our study corresponds to other pediatric reports [Campbell 2002]. In patients with accessory pathways, higher efficacy has been noted for the left-sided pathway than the right-sided pathway. The acute ablation success rate of AVNRT was $99.3 \%$, which was slightly higher than that of other pediatric reports [Kubuš 2014]. Atrioventricular block was the most common complication due to AVNRT ablation, therefore, when performing this procedure, more caution is needed and using short-term multiple discharge can reduce the probability of damage to the atrioventricular node. VT may cause significant morbidity and rarely mortality in the young [Stevenson 2007]. Elimination of the anatomic substrate by RFCA may thus offer a treatment option for symptomatic patients. In a study, reported ablation of VT in young patients achieved an acute success rate of $60 \%$ [Morwood 2004], which significantly is lower
Table 3. Acute and long-term success of RFCA for special location of AVRT and patients of different ages

\begin{tabular}{lcc}
\hline Success (\%) & Acute success & Long-term success \\
\hline Left pathway & 98.8 & 97.4 \\
Right pathway & 96.9 & 89.8 \\
$P$ value & $>.05$ & $>.05$ \\
$<3$ years old & 93.3 & 87.6 \\
$>3$ years old & 97.1 & 92.5 \\
$P$ value & $>0.05$ & $>0.05$
\end{tabular}

than the present study. Compared with VT, the acute and long-term success rate of RFCA for PVC was significantly higher, meanwhile, the recurrence rate remained low. It may be related to the complicated mechanisms of VT and difficulty of ablation. The acute ablation success rate of right ventricular outflow tract septum and left ventricular septum was the highest, followed by the free wall ventricular arrhythmias of right ventricular outflow tract. Three-dimensional cold saline perfusion ablation significantly can improve the success of ablation for PVC/VAs.

In the subgroup analysis, we explore the effect of age on RFCA and found that the acute and long-term success rate in patients $>3$ years old is slightly higher than that of the patients $<3$ years old, but it is of no statistical significance. (Table 3) The present study corresponds with other pediatric reports that showed no differences were found for success rates between infants ( $<18$ months) and noninfants [Blaufox 2001].

The major concern regarding ablation in children is the risk of complications. Small children generally are at greater risk for the procedure complications [Lenarczyk 2009; Kugler 2010]. The risk of complications in the present study is about $0.6 \%$, which is lower than other pediatric reports [Chiu 2009]. Most minor complications do not require treatment and can recover itself. Complete atrioventricular block was the most serious complication in the present study, of which 6 were treated with permanent pacemaker implantation. Other serious complications were recovered after symptomatic treatment. The occurrence of complications closely is related to the experience of the physician, and the increased experience can reduce the risk of complications.

There are several limitations to the study. First, the clinical data collected in this study span a relatively long time, and procedure-related variables such as operation proficiency, different mapping apparatus, size of catheter, and type of ablation energy may have been related to the outcome. Second, there are differences in the ablation schemes and proficiencies between different medical centers during the same period. Third, most of the duration of follow-up was less than 1 year, given the fact that certain kinds of arrhythmia showed late recurrence, a longer duration of follow-up in the future study is needed. Considering the large pediatric population in China, more and larger clinical studies are needed to further confirm the efficacy and safety of RFCA. 
CONCLUSIONS

This present study shows that RFCA is effective and safe in the treatment of arrhythmias in Chinese pediatric patients, with a high success rate exceeding $90 \%$, low recurrence rate, and it significantly reduces the risk of complications. The success rate of left-sided pathway ablation is slightly higher than that of the right-sided pathway. There is no difference in the success rate of RFCA among children of different ages. It could be the first-line therapy in the majority of pediatric patients with SVT and/or VAs.

\section{REFERENCES}

Blaufox AD, Felix GL, Saul JP. 2001. Radiofrequency Catheter Ablation in Infants $\leq 18$ Months Old When Is It Done and How Do They Fare? Short-Term Data From the Pediatric Ablation Registry. Circulation 104: 2803-2808.

Blaufox AD. 2005. Catheter ablation of tachyarrhythmias in small children. Indian Pacing Electrophysiol J 5: 51-62.

Calkins H, Yong P, Miller JM, Olshansky B, Carlson M, Saul JP et al. 1999. Catheter ablation of accessory pathways, atrioventricular nodal reentrant tachycardia, and the atrioventricular junction: final results of a prospective, multicenter clinical trial. Circulation 99: 262-770.

Campbell RM, Strieper MJ, Frias PA, Danford DA, Kugler JD. 2002. Current status of radiofrequency ablation for common pediatric supraventricular tachycardias. Journal of Pediatrics 140: 150-155.

Ceresnak SR, Dubin AM, Kim JJ, Valdes SO, Fishberger SB, Shetty I et al. 2015. Success rates in pediatric WPW ablation are improved with 3-dimensional mapping systems compared with fluoroscopy alone: a multicenter study. J Cardiovasc Electrophysiol 26: 412-416.

Chiu SN, Lu CW, Chang CW, Chang CC, Lin MT, Lin JL et al. 2009. Radiofrequency catheter ablation of supraventricular tachycardia in infants and toddlers. Circ J 73: 1717-1721.

Friedman RA, Walsh EP, Silka MJ, Calkins H, Stevenson WG, Rhodes LA et al. 2002. NASPE Expert Consensus Conference: Radiofrequency catheter ablation in children with and without congenital heart disease. Report of the writing committee North American Society of Pacing and Electrophysiology, Pacing Clin Electrophysiol 25: 1000-1017.

Johnsrude CL. 2008. Current approaches to catheter ablation for childhood tachycardia. J Ky Med Assoc. 106(5):208-217.
Kubuš P, Vít P, Gebauer RA, Zaoral L, Peichl P, Fiala M et al. 2014. Long-term results of paediatric radiofrequency catheter ablation: a population-based study. Europace 16: 1808-1818.

Kugler JD, Danford DA, Deal BJ, Gillette PC, Perry JC, Silka MJ et al. 1994. Radiofrequency catheter ablation for tachyarrhythmias in children and adolescents. The Pediatric Electrophysiologic Society. N Engl J Med 330: 1481-1487.

Lee PC, Hwang B, Chen SA, Tai CG, Chen YJ, Chiang CE et al. 2010. The results of radiofrequency catheter ablation of supraventricular tachycardia in children. Pacing Clin Electrophysiol 30: 471-481.

Lenarczyk R, Kowalski O, Pruszkowska-Skrzep P, Pluta S, Sokal A, Lenarczyk A et al. 2009. Radiofrequency catheter ablation in the treatment of arrhythmias in children efficacy, safety of the method, predictors of the procedural course and acute success. Przegl Lek 66: 418-423.

Morwood JG, Triedman JK, Berul CI, Khairy P, Alexander ME, Cecchin $\mathrm{F}$ et al. 2004. Radiofrequency catheter ablation of ventricular tachycardia in children and young adults with congenital heart disease. Heart Rhythm 1:301-308.

Rodriguez LM, Smeets JL, Timmermans C, Trappe HJ, Wellens HJ. 1997. Radiofrequency catheter ablation of idiopathic ventricular tachycardia originating in the anterior fascicle of the left bundle branch. J Cardiovasc Electrophysiol 7: 1211-1216.

Schneider HE, Kriebel T, Jung K, Gravenhorst VD, Paul T. 2010. Catheter ablation of idiopathic left and right ventricular tachycardias in the pediatric population musing noncontact mapping. Heart Rhythm 7:731-739.

Stevenson WG, Soejima K. 2007.Catheter ablation for ventricular tachycardia. Circulation 115:2750 -2760.

Strieper M, Leong T, Bajaj T, Huckaby J, Frias P, Campbell R. 2010. Does ablation of supraventricular tachycardia in children with astructurally normal heart improve quality of life? Congenit Heart Dis 5: 587-593.

Van Hare GF, Javitz H, Carmelli D, Saul JP, Tanel RE, Fischbach PS et al. 2004a. Prospective assessment after pediatric cardiac ablation: demographics, medical profiles, and initial outcomes. J Cardiovasc Electrophysiol 15: 759-770.

Van Hare GF, Javitz H, Carmelli D, Saul JP, Tanel RE, Fischbach PS et al. 2004b. Prospective Assessment after Pediatric Cardiac Ablation: Recurrence at 1 Year after Initially Successful Ablation of Supraventricular Tachycardia. Heart Rhythm 1: 188-196.

Walsh E P, Saul J P. 1991.Transcatheter ablation for pediatric tachyarrhythmias using radiofrequency electrical energy. Pediatric Annals 20: 386-388. 